\title{
Revisiting the Neutral Dynamics Derived Limiting Guanine-Cytosine Content Using the Human De Novo Point Mutation Data
}

\author{
Wentian Li $^{1}$, Yannis Almirantis ${ }^{2}$, Astero Provata ${ }^{3}$ \\ 1. The Robert S. Boas Center for Genomics and Human Genetics \\ The Feinstein Institutes for Medical Research, Northwell Health, Manhasset, NY, USA \\ 2. Theoretical Biology and Computational Genomics Laboratory, Institute of Bioscience and Applications \\ National Center for Scientific Research "Demokritos", 15341 Athens, Greece \\ 3. Statistical Mechanics and Dynamical Systems Laboratory, Institute of Nanoscience and Nanotechnology \\ National Center for Scientific Research, "Demokritos", 15341 Athens, Greece
}

\begin{abstract}
We revisit the topic of human genome guanine-cytosine content under neutral evolution. For this study, the de novo mutation data within human is used to estimate mutational rate instead of using base substitution data between related species. We then define a new measure of mutation bias which separate the de novo mutation counts from the background guaninecytosine content itself, making comparison between different datasets easier. We derive a new formula for calculating limiting guanine-cytosine content by separating CpG-involved mutational events as an independent variable. Using the formula when $\mathrm{CpG}$-involved mutations are considered, the guanine-cytosine content drops less severely in the limit of neutral dynamics. We provide evidence, under certain assumptions, that an isochore-like structure might remain as a limiting configuration of the neutral mutational dynamics.
\end{abstract}

\footnotetext{
keywords: de novo mutation, human genome, neutral evolution, limiting $\mathrm{G}+\mathrm{C}$ content, CpG dinucleotide
} 


\section{Introduction}

It is well known that in genomes of many species from fish (Costantini et al., 2007) to human (Bernardi. 1989; Costantini et al., 2006), there is an alternation of DNA segments with high and low guanine-plus-cytosine $(\mathrm{G}+\mathrm{C})$ content, called isochores (Bernardi. 1985, 2006). Such change between high and low $\mathrm{G}+\mathrm{C}$ content leads to a higher variance with respect to DNA walk properties than expected from a simple homogeneous stochastic model (Fickett et al., 1992) - thus the term heterogeneity has been adopted (Sueoka. 1962; Li et al., 1998). The physical spatial arrangement of $\mathrm{G}+\mathrm{C}$ content in genomes can be more complicated. Commonly observed examples of the spatial complexity are the variable length of these isochores, the isochore-within-isochore phenomenon, and a slower than exponential decay of base-tobase autocorrelation function, called long-range correlation (Li and Kaneko, 1992; Peng et al., 1992). An isochore is not simply a statistical signal: it has functional information also. The richest isochores tend to be gene rich (Zerial et al. 1986), and genes tend to have higher $\mathrm{G}+\mathrm{C}$ content (Clay et al., 1996). G+C content may also be associated with inner/outer region of a chromatin structure (Jabbari and Bernardi.|2017), though the cause-effect relationship between the two is not completely clear ( $\mathrm{Li} . \mid 2013)$. $\mathrm{G}+\mathrm{C}$ content of genomes of more than one thousand species is presently known (Wang, 2018).

Based on the synonymous substitution rates in protein coding regions obtained from different mammalian species, it was concluded that because $\mathrm{G} / \mathrm{C} \rightarrow \mathrm{A} / \mathrm{T}$ substitution rate (from ancestral outgroup to descendant ingroup) is higher than the $\mathrm{A} / \mathrm{T} \rightarrow \mathrm{G} / \mathrm{C}$ rate, all genomic regions will move towards a lower equilibrium $\mathrm{G}+\mathrm{C}$ content (Duret et al.. 2002; Belle et al.. 2004), though counter evidence was also presented (Alvarez-Valin et al., 2004; Gu and Li, 2006; Romiguier et al. 2010). These data from different diverging species contain information from a very long time scale, and mix various factors from mutation rates to selection.

Another observation is that the $\mathrm{G} / \mathrm{C} \rightarrow \mathrm{A} / \mathrm{T}$ to $\mathrm{A} / \mathrm{T} \rightarrow \mathrm{G} / \mathrm{C}$ substitution rate ratio is a function of the $\mathrm{G}+\mathrm{C}$ content, based on the substitutions in DNA transposons, a type of repetitive sequences (International Human Genome Sequencing Consortium, 2001). This result is not inconsistent with (Duret et al., 2002), where the absolute counts of $\mathrm{G} / \mathrm{C} \rightarrow \mathrm{A} / \mathrm{T}$ substitutions over the $\mathrm{A} / \mathrm{T} \rightarrow \mathrm{G} / \mathrm{C}$ substitution counts, increases with $\mathrm{G}+\mathrm{C}$ content. However, there is a dif- 
ference between the two approaches: the ratio in International Human Genome Sequencing Consortium. 2001) is based on conditional probability (conditional on $\mathrm{G}+\mathrm{C}$ content), whereas the ratio in (Duret et al., 2002) is unconditional. This discrepancy is not usually emphasized, which can cause confusion when different results are compared.

If we want to disentangle different causes, and only focus on the consequence of short-timescale point mutations, it would be ideal to catch mutational events in "real time". Fortunately, in the genetic study of human diseases, the "de novo" mutation - genetic variant absent in parents' genome but present in offspring's genome - has been extensively investigated in both diseased and normal samples (Kong et al.. 2012; Francioli et al.. 2015). The data becomes more important as there is evidence that de novo mutations might play a role in many human diseases, such autism spectrum disorders (Sebat et al..|2007), schizophrenia (Girard et al.. 2011), intellectual disability (Vessers et al. 2010; Hamdan et al.. 2014), developmental disorders (Deciphering Developmental Disorders Study, 2018), and others (Veltman and Brunner, 2012; Samocha et al., 2014).

There are several distinct features of de novo mutation data compared to the substitutions in mammalian species: (1) a mutation occurs within one species, the Homo sapiens, not mutations leading to base differences between species (i.e. substitution); (2) a mutation occurs in the current time, and we do not deal with ancestral mutational events in the past, which were likely to be different as it was from a potentially different environment; (3) for a normal, disease-free offspring, a de novo mutation is unlikely to be deleterious, thus the requirement of neutral evolution is satisfied. We also do not need to assume the spread as well as fixation of a mutational allele when substitutions between species are used; (4) a mutation is more directly observed, by comparing parents' and offspring's genome, not by comparing the inferred ancestral genome with the descendant genomes; (5) due to availability of the human reference genome, not only a whole genome data can be readily obtained, but also the genomic context of a mutation location is well annotated, unlike the situation of many other mammalian genomes.

The large number of de novo studies in the human genome in the recent years (Pranckeniene et al., 2018; Jonsson et al., 2018; Goldmann, 2018; Wang et al., 2019; Kessler et al., 2020) may make the data collection daunting. Fortunately, there exist de novo databases which we can use directly. Note that, unlike most people who are more interested in functional de novo muta- 
tions that cause genetic diseases, here we are interested in the neutral de novo mutations in the general population.

The study is organized as follows: we first focus on the theoretical framework relating de novo mutation rate and $\mathrm{G}+\mathrm{C}$ content. We re-derive the formula of the limiting $\mathrm{G}+\mathrm{C}$ content in single-base-mutation-driven neutral dynamics in terms of mutation counts between strong and weak bases. We define coefficients $\alpha_{1}, \alpha_{2}$ based on mutation counts (not on mutation rate), and their deviation from the value of $1 / 2$ immediately reveals which type of base will increase/decrease in the limiting dynamics. We then expand our formula to dynamics of three variables (weak bases, strong bases not-involved in $\mathrm{CpG}$ dinucleotides, and strong bases involved in $\mathrm{CpG}$ dinucleotide). We define coefficients $\beta_{1}, \beta_{2}, \beta_{3}$ based on mutation counts among these three variables, and a deviation of their value from $1 / 3$ immediately predicts the limiting dynamics of them. We carry out a data analysis using the de novo mutation database to estimate $\alpha$ 's and $\beta$ 's in different location types, as well as in different $\mathrm{G}+\mathrm{C}$ backgrounds.

\section{Theoretical formulation}

\section{Formula of limiting $\mathrm{G}+\mathrm{C}$ content based on mutation-driven neutral dynamics}

Assume that a certain number $\left(=n_{W S}\right)$ of weak $(\mathrm{A}$ or $\mathrm{T})$ to strong $(\mathrm{C}$ or $\mathrm{G})$ de novo mutation events is observed, and similarly there are $n_{S W}$ strong-to-weak mutation events. Denote the $\mathrm{G}+\mathrm{C}$-content as $x$; assume that there are $N$ genomic positions to be considered, and $M$ is the number of persons from which de novo mutation events were collected, then there are $M \cdot N \cdot x$ positions occupied by strong bases, and $M \cdot N \cdot(1-x)$ positions by weak bases. We then compose the mutation (and non-mutation) event counts matrix as:

$$
\begin{gathered}
W=A / T \\
W=A / T \\
S=C / G
\end{gathered} \quad\left(\begin{array}{cc}
M N(1-x)-n_{W S} & n_{W S} \\
n_{S W} & M N x-n_{S W}
\end{array}\right)
$$

The diagonal elements in Eq.(11) are mostly not directly counted, simply because (e.g.) $A \rightarrow A$ is not reported as a mutation event (though (e.g.) $A \rightarrow T$ is). However, we can infer them from the total number of base positions $N$, total number of samples $M$, the current $\mathrm{G}+\mathrm{C}$ 
content $x$, and the mutation counts away from the base type.

Normalizing the matrix in Eq.(1) by row sum, we obtain the conditional probability (transition probability in Markov chain):

$$
\begin{aligned}
& W=A / T \quad S=C / G \\
& \begin{array}{l}
W=A / T \\
S=C / G
\end{array} \quad\left(\begin{array}{cc}
1-\frac{n_{W S}}{M N(1-x)} & p_{W \rightarrow S} \equiv \frac{n_{W S}}{M N(1-x)} \\
p_{S \rightarrow W} \equiv \frac{n_{S W}}{M N x} & 1-\frac{n_{S W}}{M N x}
\end{array}\right)
\end{aligned}
$$

From the two weak $\leftrightarrow$ strong (conditional) transition probabilities, it is well known that the limiting G+C (strong) content is (Sueoka. 1962; Petrov and Hartle. 1999; Lynch, 2007, 2010; International Human Genome Sequencing Consortium, 2001; Li, 2011, 2013):

$$
x^{\prime}=\frac{1}{\frac{p_{S \rightarrow W}}{p_{W \rightarrow S}}+1} .
$$

An easy derivation is to consider the "detailed balance": $x^{\prime} p_{S \rightarrow W}=\left(1-x^{\prime}\right) p_{W \rightarrow S}$.

We define two new coefficients based on the mutational event counts: $\alpha_{1}=n_{S W} /\left(n_{S W}+\right.$ $\left.n_{W S}\right)$ and $\alpha_{2}=n_{W S} /\left(n_{S W}+n_{W S}\right)$. Note $\sum_{i} \alpha_{i}=1$. The conditional transition probability in Eq.(3) can be replaced by the de novo mutational event counts $\left(n_{S W}\right.$ and $\left.n_{W S}\right)$ or $\alpha$ 's:

$$
x^{\prime}=\frac{1}{\frac{n_{S W}}{n_{W S}} \cdot \frac{1-x}{x}+1} .=\frac{1}{\frac{\alpha_{1}(x)}{\alpha_{2}(x)} \cdot \frac{1-x}{x}+1} .
$$

Both total number of bases $N$ and number of persons $M$ are canceled from Eq.(4), thus we do not need to know their values. Eq. (44) shows how the limiting $\mathrm{G}+\mathrm{C}$ content ( $\mathrm{x}$ ') depends on the current $\mathrm{G}+\mathrm{C}$ content $(\mathrm{x})$, and two (actually one) mutational count based coefficients $\alpha_{1}$ $\left(\alpha_{2}=1-\alpha_{1}\right)$. Eq.(4) can be written in a more symmetric form:

$$
\frac{x^{\prime}}{1-x^{\prime}}=\frac{n_{W S}}{n_{S W}} \cdot\left(\frac{x}{1-x}\right)=\frac{\alpha_{2}(x)}{\alpha_{1}(x)} \cdot\left(\frac{x}{1-x}\right) .
$$

As long as $n_{W S}<n_{S W}$ (or $\alpha_{2}<\alpha_{1}$, more $\mathrm{C} / \mathrm{G}$ to $\mathrm{C} / \mathrm{T}$ mutation events than in the opposite direction $), x /(1-x)$ will decrease, so will $\mathrm{G}+\mathrm{C}$-content $\mathrm{x}$.

Note that Eq.(5) is not a one-time iteration, from time $t$ to time $t+1$, typically seen in the field of dynamical systems (Mav, 1976; Li and Yorke, 1975; Feigenbaum. 1978). Eq.(15) maps directly from the current $\mathrm{G}+\mathrm{C}$-content to the limiting $\mathrm{G}+\mathrm{C}$-content in one step. The base transition counts $\left(n_{S W}\right.$ and $\left.n_{W S}\right)$ or their normalized values $\left(\alpha_{1}\right.$ and $\left.\alpha_{2}\right)$ are not constant 
values, but changing as a function of the current $\mathrm{G}+\mathrm{C}$-content. If $\mathrm{G}+\mathrm{C}$-content reduces, we should also see a lower value of $\alpha_{1}$. To emphasize this point, we write this functional dependence of $\alpha_{1}$ and $\alpha_{2}$ on $x$ in Eqs.(4),5) explicitly.

In the literature, the mutational bias towards $\mathrm{W}=\mathrm{A}+\mathrm{T}$ is defined as $m=p_{S \rightarrow W} / p_{W \rightarrow S}=$ $v / u$ (Lvnch. 2007) (page-126), or the equilibrium constant in the direction of $\mathrm{W}$ base pairs $K$ (International Human Genome Sequencing Consortium, 2001) (page-886), whereas their dependence on $\mathrm{G}+\mathrm{C}$-content is not obvious. In our notation, $K=\left[\alpha_{1}(x) / \alpha_{2}(x)\right] \cdot[(1-x) / x]$ is expressed in two parts so that it is made explicit that the first part is derived purely from the mutation counts $\alpha_{1} / \alpha_{1}=n_{S W} / n_{W S}$ and the second part is unrelated to mutation counts, but purely base composition related.

There is another advantage of using $\alpha_{1,2}$ instead of the mutational bias $K$. When the values of $\alpha_{1,2}$ are compared to 0.5 , we immediately know the direction of the base type change: if $\alpha_{1}>0.5,(\mathrm{~A}+\mathrm{T})$-content will increase from the current value to its limiting value; similarly if $\alpha_{2}>0.5,(\mathrm{G}+\mathrm{C})$-content will increase. This advantage is more clear in the next subsection when three variables are considered.

\section{Formula of limiting $\mathrm{G}+\mathrm{C}$ content when $\mathrm{CpG}$ is considered separately}

Now we specifically consider a subset of strong bases within the 5'-CpG-3' dinucleotide context. The base $\mathrm{C}$ next to a base $\mathrm{G}$ in downstream (3') direction is known to have a much higher mutation rate (in particularly, to base T). The dinucleotide on the opposite strand of 5'-CpG-3' is also 5'-CpG-3', but the base $\mathrm{G}$ is expected to have a higher mutation rate. Let's denote these strong bases as $S_{p}$ ( $p$ indicates the phosphodiester bond between $\mathrm{C}$ and $\mathrm{G}$ ) and other $\mathrm{G} / \mathrm{C}$ bases not in this context as $S_{n}$. We also assume among strong $\mathrm{G} / \mathrm{C}$ bases, a proportion of $y$ of them are in $S_{p}$. Though not common, it is still possible to have a mutation from one $S_{p}$ base to another $S_{p}$ base, e.g., $5^{\prime}-C G G-3^{\prime} \rightarrow 5^{\prime}-C C G-3^{\prime}$. 
Similar to Eq.(1), the number of mutation counts for three types of base (W, $\left.S_{n}, S_{p}\right)$ are:

$$
\left.\begin{array}{cccc} 
& W & S_{n} & S_{p} \\
W & \left(M N(1-x)-n_{W S n}-n_{W S p}\right. & n_{W S n} & n_{W S p} \\
S_{n} & n_{S n W} & M N x(1-y)-n_{S n W}-n_{S n S p} & n_{S n S p} \\
S_{p} & n_{S p W} & n_{S p S n} & M N x y-n_{S p W}-n_{S p S n}
\end{array}\right)
$$

and again, the row normalized matrix is the transition matrix:

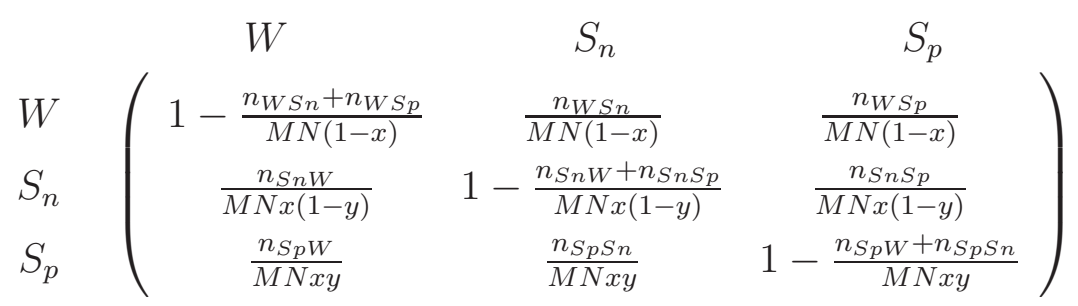

The limiting composition of $W, S_{n}, S_{p}$ is proportion to the eigenvector of the transpose (switching rows and columns) of Eq.([6) corresponding to the eigenvalue equal to 1 (which is the largest eigenvalue of a Markov transition matrix) (see Appendix). We obtain such a (unnormalized) eigenvector for the transpose of Eq.(6]) by Wolfram Alpha (www.wolframalpha.com) as:

$$
\left(\begin{array}{c}
\left(n_{S p W} n_{S n W}+n_{S n S p} n_{S p W}+n_{S p S n} n_{S n W}\right)(1-x) \\
\left(n_{W S n} n_{S p S n}+n_{W S p} n_{S p S n}+n_{S p W} n_{W S n}\right) x(1-y) \\
\left(n_{W S p} n_{S n S p}+n_{W S n} n_{S n S p}+n_{S n W} n_{W S p}\right) x y
\end{array}\right) \propto\left(\begin{array}{c}
\beta_{1}(x) \cdot(1-x) \\
\beta_{2}(x) \cdot x(1-y) \\
\beta_{3}(x) \cdot x y
\end{array}\right) .
$$

Although Eq.(7) looks complicated, it can be memorized by the illustration in Fig.1. Note that again the genome size $\mathrm{N}$ and number of persons $\mathrm{M}$ are not present in the limiting composition formula Eq.(17).

We introduce new coefficients $\beta_{i}(\mathrm{i}=1,2,3)$ to be proportional to the coefficients in the left column-array in Eq.(17), but normalized (i.e., divided by the sum of all products of two transition counts). Note that $\sum_{i} \beta_{i}=1$ doesn't mean the right column array itself in Eq.(7) is normalized. Our introduction of $\beta_{i}$ coefficients will make the comparison between different data easier, because they are based purely on mutational counts. Also, if $\beta_{1}=\beta_{2}=\beta_{3}=1 / 3$ implies no change in the composition of weak, CpG-unrelated-strong, and CpG-related-strong base types, and deviation of $\beta$ from $1 / 3$ easily points to the direction of change in the composition. To emphasize the fact that $\left\{\beta_{i}\right\}$ are not constant in the dynamics, we write their dependence on $\mathrm{G}+\mathrm{C}-$ content explicitly in Eq.(7). 


\section{Data analyses}

\section{Filtering neutral de novo mutation events}

We use the denovo-db v1.6.1 (http://denovo-db.gs.washington.edu/denovo-db/, August 19, 2018). The files denovo-db.ssc-samples.variants.tsv and denovo-db.non-ssc-samples.variants.tsv are used. Each line in these files is a mutational event in a person with a particular annotation. Therefore, for a mutation in a coding region with multiple transcripts, each mutation event may occupy multiple lines. There are 628,234 lines in the two files. The genomic coordinates are in hg19/GRCh37.

We filter the de novo mutations by the following criteria: (1) The mutation is a bi-allelic single-nucleotide-polymorphism (SNP); (2) The person's phenotype is a normal "control"; (3) Y-chromosome variants are excluded; (4) the base is consistent with the reference genome of hg19/GRCh37. The criterion \#1 serves to avoid the more complicated mutational events such as indels and multi-allelic variants whose detection is less reliable. The criteria \#2 is to make sure that the mutational event is neutral, and less likely to be deleterious. These filterings reduce the number of lines to 110,989 .

The following studies have contributed the most to the de novo mutation event counts: Turner2017 (83187, 75.0\%) (Turner et al. |2017), GONL (15896, 14.3\%) (Genome of the Netherlands Consortiu 2014), Turner2016 (3541, 3.2\%) (Turner et al..|2016), Iossifov2014 (3521, 3.2\%) (Iossifov et al.. 2014), Werling2018 (3302, 3.0\%) (Werling et al., 2018), Krumm (1014, 0.9\%) (Krumm et al.. 2015), Yuen2017 (181, 0.16\%) (Yuen et al., 2017), Gulsuner2013 (170, 0.15\%) (Gulsuner et al.. 2013), Conrad2011 (59, 0.05\%) (Conrad et al. 2011), Besenbacher2014 (52,0.047\%) (Besenbacher et al.. 2015), Rauch2012 (509, 0.045\%) (Rauch et al.. 2012), and ASD3 (15, 0.014\%).

Besides the information provided by denovo-db, we have added these extra information by using the hg19/GRCh37 reference genome: (1) $\mathrm{G}+\mathrm{C}$ base and CpG dinucleotide count of $2 \mathrm{~kb}$ window centered at the SNP; (2) $\mathrm{G}+\mathrm{C}$ base and $\mathrm{CpG}$ dinucleotide count of 20kb window centered at the SNP; (3) the triplet context of the SNP; and (4) the triplet context after the mutation.

The denovo-db provides 18 location-types which we condense to 9 types: intron (and intronnear-splice): 58824 lines, intergenic: 40375 lines, upstream-gene and downstream-gene: 4832 
lines, missense(and missense-near-splice): 3235 lines, 5' and 3' UTR: 1514 lines, synonymous (and synonymous-nea-splice): 1336 lines, non-coding-exon (and non-coding-exon-near-splice): 634 lines, stop-lost and stop-gain: 149 lines, splice-acceptor and splice-donor: 81 lines.

Fig 2 shows the distribution of CADD (combined annotation dependent depletion) value (Kircher et al.. 2014), percentage of non-repetitive-sequence (uppercase letter), 2kb window $\mathrm{G}+\mathrm{C}$ content, 20kb window $\mathrm{G}+\mathrm{C}$ content, $2 \mathrm{~kb}$ widow $\mathrm{CpG} \% /(\mathrm{G}+\mathrm{C}) \%$, and 20kb widow $\mathrm{CpG} \% /(\mathrm{G}+\mathrm{C}) \%$ of all these 9 location types. Most of the result in Fig,2 is known. For example, the functional impact of variants is the highest for stop-gain/lost, followed by missense; intergenic regions contain more repetitive sequences or transposons; genic regions can be of high- $(\mathrm{G}+\mathrm{C})$-content; etc. We further show that larger window (20kb) statistics have more narrow distributions, and intron regions (even more so than intergenic regions) avoid $\mathrm{CpG}$ dinucleotides.

\section{De novo mutation derived $\alpha$ and $\beta$ coefficients}

Table 1 shows the raw count of different types of de novo mutations in 9 different variant types described in the last section. The two $\alpha_{1}$ and $\alpha_{2}$ coefficients for the two-base type model and three $\beta_{1}, \beta_{2}, \beta_{3}$ coefficients for the three-base type model are listed in Table 1. Although we cannot assume neutral dynamics for variants in the functional categories, whether an $\alpha_{i}$ coefficient larger or smaller than $1 / 2$, and whether a $\beta_{i}$ coefficient larger or smaller than $1 / 3$ will indicate which direction the mutational force is pushing. In all functional categories, W $(\mathrm{A}+\mathrm{T})$ base content will be pushed higher by mutation, $\mathrm{S}(\mathrm{G}+\mathrm{C})$ and $\mathrm{CpG}$ content will be pushed lower. The stop-gain/loss and splice acceptor/donor categories contain very few de novo mutation counts. However, the mutational force would drive the $\mathrm{CpG}$ content higher in splice sites, while deplete $\mathrm{CpG}$ from stop sites.

Bases in the intergenic regions can be assumed to follow a neutral evolution without constraints. We further partition the de novo mutational events in intergenic regions according to its surrounding $(2 \mathrm{~kb})(\mathrm{G}+\mathrm{C})$-content, and $\alpha, \beta$ coefficients are calculated in each $(\mathrm{G}+\mathrm{C})$ content quantile. The results are shown in Table 2, We can see that not only $\alpha_{1}>1 / 2$ and $\beta_{1}>$ $1 / 3$, but also their values increase with the surrounding $\mathrm{G}+\mathrm{C}$ content. This result is consistent 
with previous publications (Duret et al. 2002; International Human Genome Sequencing Consortium. 2001). Table 2 also shows that $\beta_{3}<1 / 3$, and decreases with surrounding $\mathrm{G}+\mathrm{C}$ content. Dependence of $\mathrm{CpG}$ mutation rate on local $\mathrm{G}+\mathrm{C}$ content has also been reported before (Fryxell and Moon, 2005).

\section{Evidence of two different limiting $\mathrm{G}+\mathrm{C}$ contents}

To further examine the prediction of our neutral mutational dynamics, using the mutation rates based on the de novo mutational event count, as a function of current $\mathrm{G}+\mathrm{C}$ content, we expand the previous six $\mathrm{G}+\mathrm{C}$ content quantiles to eight, with the highest $\mathrm{G}+\mathrm{C}$ range split into three more $\mathrm{G}+\mathrm{C}$ regions. This partition would lead to around $6000-7000$ intergenic de novo mutational events in each one of the lower $\mathrm{G}+\mathrm{C}$ brackets, but 2000-3000 intergenic mutational events in the last three high $\mathrm{G}+\mathrm{C}$ brackets. The mutation counts of various types,

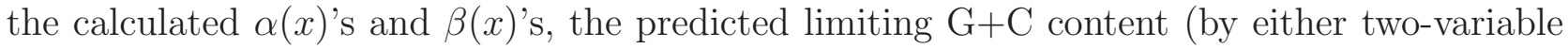
or three-variable equation) and limiting $\mathrm{CpG} /(\mathrm{G}+\mathrm{C})$, are shown in Table 2, The current intergenic $\mathrm{G}+\mathrm{C}$ content and $\mathrm{CpG} /(\mathrm{G}+\mathrm{C})$ values, calculated directly from the hg19 intergenic sequences (an intergenic sequence longer than 10kb is partitioned into pieces of 10kb length), are shown in Fig 3. We notice that $\mathrm{CpG} /(\mathrm{G}+\mathrm{C})$ is positively correlated with $(\mathrm{G}+\mathrm{C}) \%$, as it involves the product of two strong bases.

Fig 4 depicts the mutational data as a function of current $(\mathrm{G}+\mathrm{C}) \%$ from various perspectives. Fig $4(\mathrm{~A})$ shows that the $p(S \rightarrow W) / p(S \rightarrow W)$ is not constant, but decreases at high $(\mathrm{G}+\mathrm{C})$ content. By the prediction in Eq.(3) , the limiting $(\mathrm{G}+\mathrm{C}) \%$ will be higher for the current $(\mathrm{G}+\mathrm{C})$-rich intergenic regions, as shown in Fig $4(\mathrm{~B})$. Interestingly, the three-variable prediction (by considering $\mathrm{CpG}$-containing strong base as a distinct variable) leads to slightly higher limiting $(\mathrm{G}+\mathrm{C}) \%$ than the two-variable prediction. Fig $4(\mathrm{C})$, however, shows that the limiting $\mathrm{CpG} /(\mathrm{G}+\mathrm{C})$ may not be off very much from the current $\mathrm{CpG} /(\mathrm{G}+\mathrm{C})$ value. Fig 4 (D) is yet another way to look at the same data. If the mutational rate is the same in regions with different current $(\mathrm{G}+\mathrm{C}) \%$, the proportion of $\mathrm{G}$ or $\mathrm{C}$ bases among those that experience a de novo mutation will be linearly proportional to the current $(\mathrm{G}+\mathrm{C}) \%$. Nonlinearity of this "substituted-base G+C content" in bacterial genomes has been observed (Bohlin et al., 2018). 
The fact that our Fig,4(D) is further away from the diagonal line (see the grey dashed line) indicates that human genome is not in a base composition equilibrium state as in bacterial genomes.

The results in Fig 4 may indicate that isochores are maintained by neutral mutational dynamics if the mutational rate is estimated from the de novo mutational events. However, there are still two possibilities: (1) the relatively low mutational AT-driving-force observed in the current high $\mathrm{G}+\mathrm{C}$ region is supposed to be still low when the $\mathrm{G}+\mathrm{C}$ content in the same region is lower in time. We may justify this assumption by a hypothesis that the mutation rate in this region is perhaps determined by the three-dimensional chromatin structure than by the $\mathrm{G}+\mathrm{C}$ content. (2) our high $\mathrm{G}+\mathrm{C}$ intergenic region might be embedded in high $\mathrm{G}+\mathrm{C}$ genic regions which protect the $\mathrm{G}+\mathrm{C}$ decay by selection force. In that case, the relatively low AT-driving-force in the intergenic region is not really neutral. The limiting isochore conclusion may not be reached if we assume that the mutational driving force in the current high $\mathrm{G}+\mathrm{C}$ regions becomes stronger with time, due to the lower $\mathrm{G}+\mathrm{C}$ content in the future. However, there is no way to prove this with the current data.

\section{Discussion}

In this study, we re-visit the topic which was popular in the past, on base composition change (Duret et al., 2002; Gu and Li, 2006; Alvarez-Valin et al., 2004; Romiguier et al., 2010), but focus one one species only, the homo sapiens. Towards this, we rely on the mutational events observed in human only, i.e., the de novo mutation by comparing the genomic sequences between parents and offspring. A reliable data on both the mutation rate and context-dependence is clearly important. Previous work calculated this information by comparing the orthologous regions between human and chimpanzee, considering chimp as ancestral and count any single nucleotide change from chimp to human as mutational events (Supplementary text of (Samocha et al.. 2014)). This approach may obtain more counts, but the directionality of the mutational events can be questioned. The data we use is guaranteed for the mutational direction (from parents to offspring) which is an important piece of information on context analysis. 
Rare variants might be another type of data to study mutation rates and context effect (Chakraborty. 1981; Kimura. 1983; Neel et al., 1986). However, this approach should deal with sequencing errors and private variant (i.e., variant found only in one person) should be validated. Also, population specific reference genomes should be available, so that a so-called rare variant according to the standard reference genome might be not so rare in a particular population, and multiple mutations on the same site should be corrected. Considering the importance of estimating the background neutral mutational rate in the assessment of excess mutation in a particular gene (Samocha et al., 2014), it could be interesting to compare different approaches, with the anticipation of further complexity due to factors such as gender and age (Jonsson et al., 2018), population (Mathieson and Reich, 2017; Narasimhan et al.. 2017), and chromosome regions (Harpak et al., 2016).

Our conclusion that isochore-like structure, i.e., different regions having different $\mathrm{G}+\mathrm{C}$ contents, can be maintained in the limiting configuration of the neutral dynamics, has already been implied in (page 886 of) (International Human Genome Sequencing Consortium, 2001): "if $\mathrm{K}$ is the equilibrium constant ... then the equilibrium GC content should be $1 /(1+\mathrm{K}) \ldots$ $(\mathrm{K})$ varies as a function of local GC content". Because the currently $(\mathrm{G}+\mathrm{C})$-rich regions have lower $\mathrm{K}$ (equivalent to our $\left[\alpha_{1}(x) / \alpha_{2}(x)\right] \cdot[(1-x) / x]$ in Eq.(44)), they should also have a higher $\mathrm{G}+\mathrm{C}$ content in the limiting equilibrium state. However, our conclusion is reached based on a more realistic three-variable dynamics (Eq.7). We also caution on an assumption required for reaching this conclusion, i.e. the mutational rate is a chromosome regional property and may not be a property of $\mathrm{G}+\mathrm{C}$ content itself. To confirm or reject the assumption, it might be necessary to follow the temporal base composition dynamics in an intergenic $\mathrm{G}+\mathrm{C}$ rich region in the human genome.

\section{Acknowledgment}

W.L. thanks the financial support from the Robert Boas Center for Genomics and Human Genetics. 


\section{Appendix}

\section{A Derivation of the limiting composition based on mutation rate}

The master equation or continuous time Markov process for the dynamics of a genomic unit with multiple $(m)$ states is (superscript $T$ is for transpose):

$$
\frac{d \overrightarrow{\mathbf{P}}}{d t}=\left(\mathbf{M}^{\mathbf{T}}-\mathbf{I}\right) \overrightarrow{\mathbf{P}}
$$

where $\overrightarrow{\mathbf{P}}$ is the composition array with $m$ elements, and $\mathbf{M}_{m \times m}=\left\{M_{i j}\right\}=\left\{P_{i \rightarrow j}\right\}(i, j=$ $1 \ldots m)$ is the $m \times m$ transition matrix, with $P_{i \rightarrow j}$ the unit time probability for state $i$ to change to state $j$, and $\mathbf{I}$ the $m \times m$ identity matrix. The value of $m$ is 4 for nucleotide bases, 16 for dinucleotides, 20 for amino acids, 64 for codons, and any values in between or beyond when degenerate/equivalent states of the genomic unit are combined. For example, if the strand symmetry is considered, $m=2$; if $\mathrm{A}$ and $\mathrm{T}$ are combined into weak and $\mathrm{C}$ and $\mathrm{G}$ combined to strong, $m=2$; if $\mathrm{C}$ or $\mathrm{G}$ within dinucleotide $\mathrm{CpG}$ is distinguished from not within, $m=3$; and if stop codons are excluded from all codons, $m=21$, etc.

Eq.(8) can be derived by examining the source of state- $j$ frequency change at time $t+d t$ from that at time $t$ :

$$
\begin{aligned}
p_{j}(t+d t) & =p_{j}(t)+\sum_{i \neq j} p_{i}(t) P_{i \rightarrow j} d t-p_{j}(t) \sum_{k \neq j} P_{j \rightarrow k} d t \\
& =p_{j}(t)+\sum_{i \neq j} p_{i}(t) P_{i \rightarrow j} d t-p_{j}(t)\left(1-P_{j \rightarrow j}\right) d t \\
& =p_{j}(t)+\sum_{i} p_{i}(t) P_{i \rightarrow j} d t-p_{j}(t) d t
\end{aligned}
$$

then,

$$
\begin{aligned}
\frac{p_{j}(t+d t)-p_{j}(t)}{d t} & =\sum_{i} p_{i}(t) P_{i \rightarrow j}-p_{j}(t) \\
& =\sum_{j^{\prime}} P_{j^{\prime} \rightarrow i^{\prime}} p_{j^{\prime}}(t)-p_{j}(t) \\
& =\sum_{j^{\prime}} \mathbf{M}_{i^{\prime} j}^{T} p_{j^{\prime}}(t)-p_{j}(t)
\end{aligned}
$$

which is Eq.(8) in the $d t \rightarrow 0$ limit. 
The equilibrium composition is the solution of $d \tilde{\mathbf{P}} / d t=0=\left(\mathbf{M}^{\mathbf{T}}-\mathbf{I}\right) \overrightarrow{\mathbf{P}}$ which is an eigenvalue/eigenvector problem. This type of dynamical systems is also called (multi) compartmental systems (Jacquez, 1972), and it is known that the only non-negative eigenvalue of compartmental matrix $\mathbf{M}^{\mathbf{T}}-\mathbf{I}$ is zero (the largest eigenvalue of matrix $\mathbf{M}^{\mathbf{T}}$ is one) (Jacquez, 1972). In other words, the limiting composition is the (normalized) eigenvector corresponding to the eigenvalue $=1$ of the transpose of Markov transition matrix. 


\section{References}

F Alvarez-Valin, O Clay, S Cruveiller, G Bernardi (2004), Inaccurate reconstruction of ancestral GC levels creates a "vanishing isochores effect, Mol. Phylogenet. and Evol., 31:788-793.

EMS Belle, L Duret, N Gatlier, A Eyre-Walker (2004), The decline of isochores in mammals: an assessment of the GC content variation along the mammalian phylogeny, J. Mol. Evol., 58:653-660.

G Bernardi (1989), The isochore organization of the human genome, Ann. Rev. Genet., 23:637-661.

G Bernardi (2006), Structural and Evolutionary Genomics: Natural Selection in Genome Evolution (Elsevier).

G Bernardi, B Olofsson, J Filipski, M Zerial, J Salinas, G Cuny, M Meunier-Rotival, F Rodier (1985), The mosaic genome of warm-blooded vertebrates, Science, 228:953-958.

S Besenbacher, S Liu, JM Izarzugaza, J Grove, K Belling, J Bork-Jensen, S Huang, TD Als, S Li, R Yadav, et al. (2015), Novel variation and de novo mutation rates in population-wide de novo assembled Danish trios, Nature Commun., 6:5969.

J Bohlin, V Eldholm, O Brynildsrud, JH Petterson, K Alfsnes (2018), Modeling of the GC content of the substituted bases in bacterial core genomes, BMC Genomics, 19:589.

R Chakraborty (1981), Estimation of mutation rates from the number of rare alleles in a sample, Ann. Hum. Biol., 8:221-230.

O Clay, S Cacció, S Zoubak, D Mouchiroud, G Bernardi (1996), Human coding and noncoding DNA: compositional correlations, Mol. Phylogenet. and Evol., 5:2-12.

DF Conrad, JE Keebler, MA DePristo, SJ Lindsay, Y Zhang, F Casals, Y Idaghdour, CL Hartl, C Torroja, KV Garimella, M Zilversmit, R Cartwright, GA Rouleau, M Daly, EA Stone, ME Hurles, P Awadalla, 1000 Genomes Project (2011), Variation in genome-wide mutation rates within and between human families, Nature Genet., 43:712-714.

M Costantini, F Auletta, G Bernardi (2007), Isochore patterns and gene distributions in fish genomes, Genomics, 90:364-371.

M Costantini, O Clay, F Auletta, G Bernardi (2006), An isochore map of human chromosomes, Genome Res., $16: 536-541$.

Deciphering Developmental Disorders Study (2018), Prevalence and architecture of de novo mutations in developmental disorders, Nature, 542:433-438. 
L Duret, M Semon, G Piganeau, D Mouchiroud, N Galtier (2002), Vanishing GC-rich isochores in mammalian genomes, Genetics, 162:1837-1847.

MJ Feigenbaum (1978), Quantitative universality for a class of nonlinear transformations, J. Stat. Phys., 19:25-52.

JW Fickett, DC Torney, DR Wolf (1992), Base compositional structure of genomes, Genomics, 13:1056-1064.

LC Francioli, PP Polak, A Koren, A Menelaou, S Chun, I Renkens, Genome of the Netherlands Consortium, CM van Duijn, M Swertz, C Wijmenga, et al. (2015), Genome-wide patterns and properties of de novo mutations in humans, Nature Genet., 47:822-826.

KJ Fryxell and WJ Moon (2005), CpG mutation rates in the human genome are highly dependent on local GC content, Mol. Biol. and Evol., 22:650-658.

Genome of the Netherlands Consortium (2014), Whole-genome sequence variation, population structure and demographic history of the Dutch population, Nature Genet., 46:818-825.

SL Girard, J Gauthier, A Noreau, L Xiong, S Zhou, L Jouan, A Dionne-Laporte, D Spiegelman, E Henrion, O Diallo, P Thibodeau, I Bachand, JYJ Bao, AHY Tong, CH Lin, B Millet, N Jaafari, R Joober, PA Dion, S Lok, MO Krebs, GA Rouleau (2011), Increased exonic de novo mutation rate in individuals with schizophrenia, Nature Genet., 43:860863.

JM Goldmann (2018), Characterization of de novo mutations in the human germline (Ph.D Thesis, Dept of Human Genetics, Radboud University Medical Center).

J Gu and WH Li (2006), Are GC-rich isochores vanishing in mammals? Gene, 385:50-56.

S Gulsuner, T Walsh, AC Watts, MK Lee, AM Thornton, S Casadei, C Rippey, H Shahin, Consortium on the Genetics of Schizophrenia (COGS), PAARTNERS Study Group, (2013), Spatial and temporal mapping of de novo mutations in schizophrenia to a fetal prefrontal cortical network, Cell, 154:518-529.

FF Hamdan, M Srour, JM Capo-Chichi, H Daoud, C Nassif, L Patry, C Massicotte, A Ambalavanan, D Spiegelman, O Diallo, E Henrion, A Dionne-Laporte, A Fougerat, AV Pshezhetsky, S Venkateswaran, GA Rouleau, JL Michaud (2014), De novo mutations in moderate or severe intellectual disability, PLOS Genet., 10:1004772.

A Harpak, A Bhaskar, JK Pritchard (2016), Mutation rate variation is a primary determinant of the distribution of allele frequencies in humans, PLoS Genet., 12:e1006489.

International Human Genome Sequencing Consortium (2001), Initial sequencing and analysis of the human genome, Nature, 409:860-921. 
I Iossifov, BJ O’Roak, SJ Sanders, M Ronemus, N Krumm, D Levy, HA Stessman, KT Witherspoon, L Vives, KE Patterson, et al. (2014), The contribution of de novo coding mutations to autism spectrum disorder, Nature, 515:216-221.

K Jabbari and G Bernardi (2017), An isochore framework underlies chromatin architecture, PLoS ONE, 12:e0168023.

JA Jacquez (1972), Compartmental Analysis in Biology and Medicine: Kinetics of Distribution of TracerLabeled Materials (Elsevier).

H Jónsson, P Sulem, GA Arnadottir, Pálsson, HP Eggertsson, S Kristmundsdottir, F Zink, B Kehr, KE Hjorleifsson, B Jensson, et al. (2018), Multiple transmissions of de novo mutations in families, Nature Genet., 50:1674-1680.

MD Kessler, DP Loesch, JA Perry, NL Heard-Costa, D Taliun, BE Cade, H Wang, M Daya, J Ziniti, S Datta, et al. (2020), De novo mutations across 1465 diverse genomes reveal mutational insights and reductions in the Amish founder population, Proc. Natl. Acad. Sci., 117:2560-2569.

M Kimura (1983), Rare variant alleles in the light of the neutral theory, Mol. Biol. and Evol., 1:84-93.

M Kircher, DM Witten, P Jain, BJ O'Roak, GM Cooper, J Shendure (2014), A general framework for estimating the relative pathogenicity of human genetic variants, Nature Genet., 46:310315.

A Kong, ML Frigge, G Masson, S Besenbacher, P Sulem, G Magnusson, SA Gudjonsson, A Sigurdsson, A Jonasdottir, A Jonasdottir, (2012), Rate of de novo mutations and the importance of fathers age to disease risk, Nature, 488:471-475.

N Krumm, TN Turner, C Baker, L Vives, K Mohajeri, K Witherspoon, A Raja, BP Coe, HA Stessman, ZX He, et al. (2015), Excess of rare, inherited truncating mutations in autism, Nature Genet., 47:582-588.

TY Li and JA Yorke (1975), Period three implies chaos, Am. Math. Monthly, 82:985-992.

W Li (2011), On parameters of the human genome, Journal of Theoretical Biology, 288:92-104.

W Li (2013), G+C content evolution in the human genome, eLS (John Wiley \& Sons, Ltd: Chichester). doi: 10.1002/9780470015902.a0021751

W Li and K Kaneko (1992), Long-range correlation and partial 1/f spectrum in a non-coding DNA sequence, Europhys. Lett., 17:655-660.

W Li, G Stolvitzky, P Bernaola-Galvan, JL Oliver (1998), Compositional heterogeneity within, and uniformity between, DNA sequences of yeast chromosomes, Genome Res., 8:916-928. 
M Lynch (2007), The Origins of Genome Architecture (Sinauer Associations, Inc.: Sunderland, MA).

M Lynch (2010), Rate, molecular spectrum, and consequences of human mutation, Proc. Natl. Acad. Sci., 107:961-968.

RM May (1976), Simple mathematical models with very complicated dynamics, Nature. 261:459467.

VM Narasimhan, R Rahbari, A Scally, A Wuster, D Mason, Y Xue, J Wright, RC Trembath, ER Maher, DA van Heel, A Auton, ME Hurles, C Tyler-Smith, R Durbin (2017), Estimating the human mutation rate from autozygous segments reveals population differences in human mutational processes, Nature Comm., 8:303.

I Mathieson and D Reich (2017), Differences in the rare variant spectrum among human populations, PLoS Genet., 13:e1006581.

JV Neel, HW Mohrenweiser, ED Rothman, JM Naidu (1986), A revised indirect estimate of mutation rates in Amerindians, Am. J. Hum. Genet., 38:649-666.

CK Peng, SV Buldyrev, AL Goldberger, S Havlin, F Sciortino, M Simons, HE Stanley (1992), Long-range correlations in nucleotide sequences, Nature, 356168-170.

DA Petrov and DL Hartle (1999), Patterns of nucleotide substitution in Drosophila and mammalian genomes, Proc. Natl. Acad. Sci., 96,14751479.

L Pranckènienè, A Jakaitienè, L Ambrozaitytè, I Kavaliauskienè, V Kučinskas Insight into de novo mutation variation in Lithuanian exome, Front. Genet., 9:315.

A Rauch, D Wieczorek, E Graf, T Wieland, S Endele, T Schwarzmayr, B Albrecht, D Bartholdi, J Beygo, N Di Donato, et al. (2012), Range of genetic mutations associated with severe non-syndromic sporadic intellectual disability: an exome sequencing study, Lancet, 380:1674-1682.

J Romiguier, V Ranwez, EJP Douzery, N Galtier (2010), Contrasting GC-content dynamics across 33 mammalian genomes: Relationship with life-history traits and chromosome sizes, Genome Res., 20:1001-1009.

K E Samocha, EB Robinson, SJ Sanders, C Stevens, A Sabo, LM McGrath, JA Kosmicki, K Rehnström, S Mallick, A Kirby, et al. (2014), A framework for the interpretation of de novo mutation in human disease, Nature Genet., 46:944-950.

J Sebat, B Lakshmi, D Malhotra, J Troge, C Lese-Martin, T Walsh, B Yamrom, S Yoon, A Krasnitz, J Kendall, et al. (2007), Strong association of de novo Copy number mutations with autism, Science, 316:445-449.

N Sueoka (1962), On the genetic basis of variation and heterogeneity of DNA base composition, Proc. Natl. Acad. Sci., 48:582-592. 
TN Turner, F Hormozdiari, MH Duyzend, SA McClymont, PW Hook, I Iossifov, A Raja, C Baker, K Hoekzema, HA Stessman, et al. (2016), Genome sequencing of autism-affected families reveals disruption of putative noncoding regulatory DNA, Am. J. Hum. Genet., 98:58-74.

TN Turner, BP Coe, DE Dickel, K Hoekzema, BJ Nelson, MC Zody, ZN Kronenberg, F Hormozdiari, A Raja, LA Pennacchio, RB Darnell, EE Eichler (2017), Genomic patterns of de novo mutation in simplex autism, Cell, 171:710-722.

JA Veltman and HG Brunner (2012), De novo mutations in human genetic disease, Nature Rev. Genet., $13: 565-575$.

LE Vissers, J de Ligt, C Gilissen, I Janssen, M Steehouwer, P de Vries, B van Lier, P Arts, N Wieskamp, M del Rosario, BW van Bon, A Hoischen, BB de Vries, HG Brunner, JA Veltman (2010), A de novo paradigm for mental retardation, Nature Genet., 42:1109-1112.

D Wang (2018), GCevobase: an evolution-based database for GC content in eukaryotic genomes, Bioinformatics, 34:2129-2131.

W Wang, R Coronminas, GN Lin (2019), De novo mutations from whole exome sequencing in neurodevelopmental and psychiatric disorders: from discovery to application, Front. Genet., 10:258.

DM Werling, H Brand, et al. (2018), An analytical framework for whole-genome sequence association studies and its implications for autism spectrum disorder, Nature Genet., 50:727-736.

RKC Yuen, D Merico, et al. (2017), Whole genome sequencing resource identifies 18 new candidate genes for autism spectrum disorder, Nature Neurosci., 20:602-611.

M Zerial, J Salinas, J Filipski, G Bernardi (1986), Gene distribution and nucleotide sequence organization in the human genome, Eru. J. Biochem., 160:479-485. 


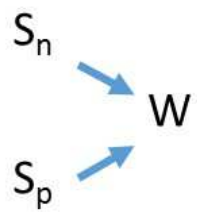

(1)

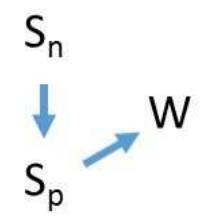

(2)

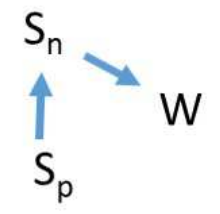

(3)

Figure 1: Illustration of the analytic expression of the first element in the limiting array in Eq.(17), which is proportional to $\beta_{1}$. This expression is the sum of three terms: $n_{S p W} n_{S W}, n_{S n S p} n_{S p W}$, and $n_{S p S n} n_{S n W}$ where $W$ is weak base (A or T), $S_{n}$ is strong base (C or G) not involved in a CpG context, and $S_{p}$ for $\mathrm{S}$ in a $\mathrm{CpG}$ context. These three terms can be represented by the subplots (1), (2) and (3) . 

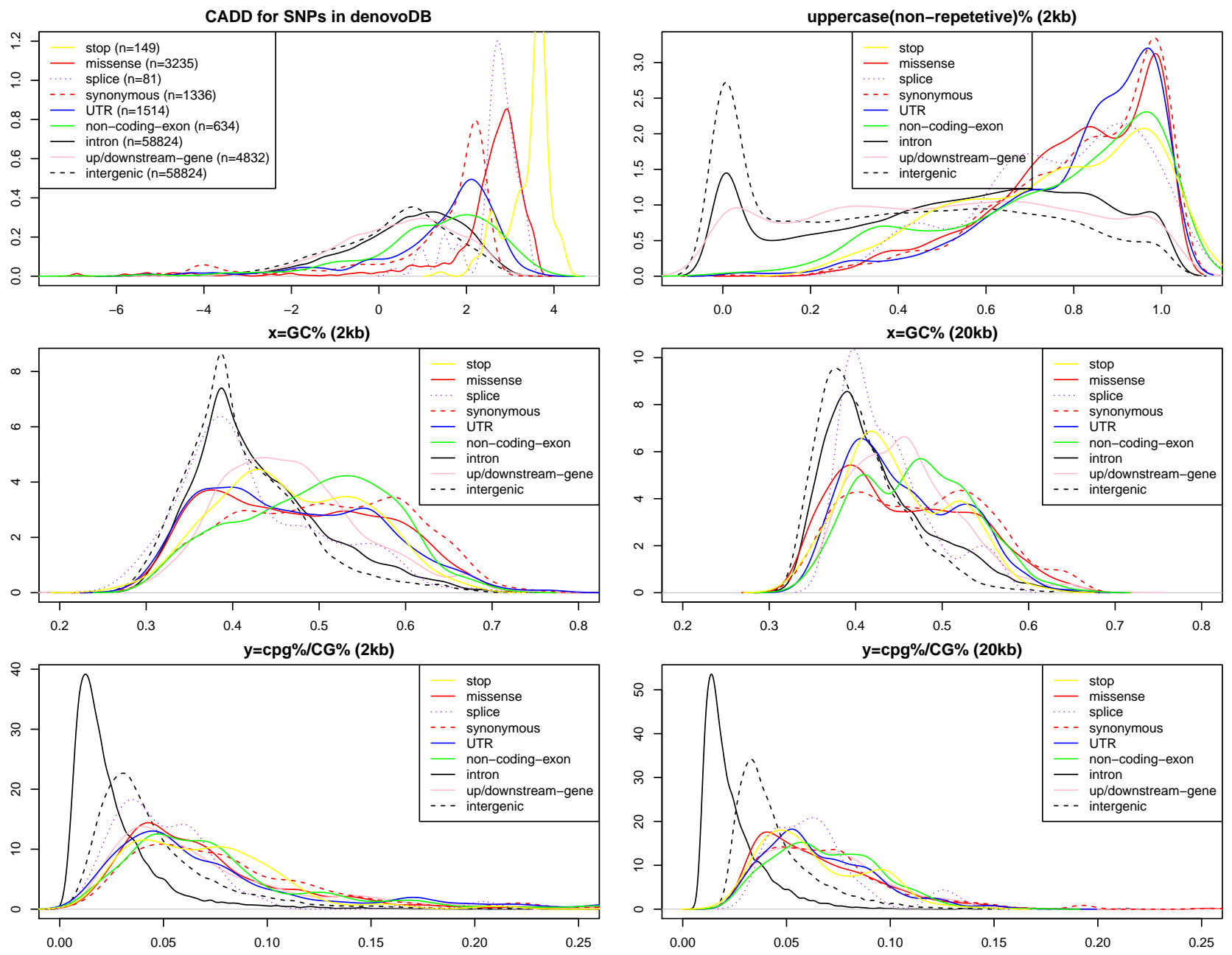

Figure 2: Distribution of various statistics of the de novo mutations according to nine different categories: stopgain/loss, missense, splice donor/acceptor, synonymous, 3'/5'-UTR, non-coding-exon, intron, up/downstreamgene, intergenic. (1) CADD; (2) percentage of non-repetitive sequence in the $2 \mathrm{~kb}$ window centered at the mutation site ; $(3)(\mathrm{G}+\mathrm{C})$-content in the $2 \mathrm{~kb}$ window; (4) $(\mathrm{G}+\mathrm{C})$-content in the $20 \mathrm{~kb}$ window; (5) $\mathrm{CpG} /(\mathrm{G}+\mathrm{C})$ in the $2 \mathrm{~kb}$ window; $(6) \mathrm{CpG} /(\mathrm{G}+\mathrm{C})$ in the $20 \mathrm{~kb}$ window. 


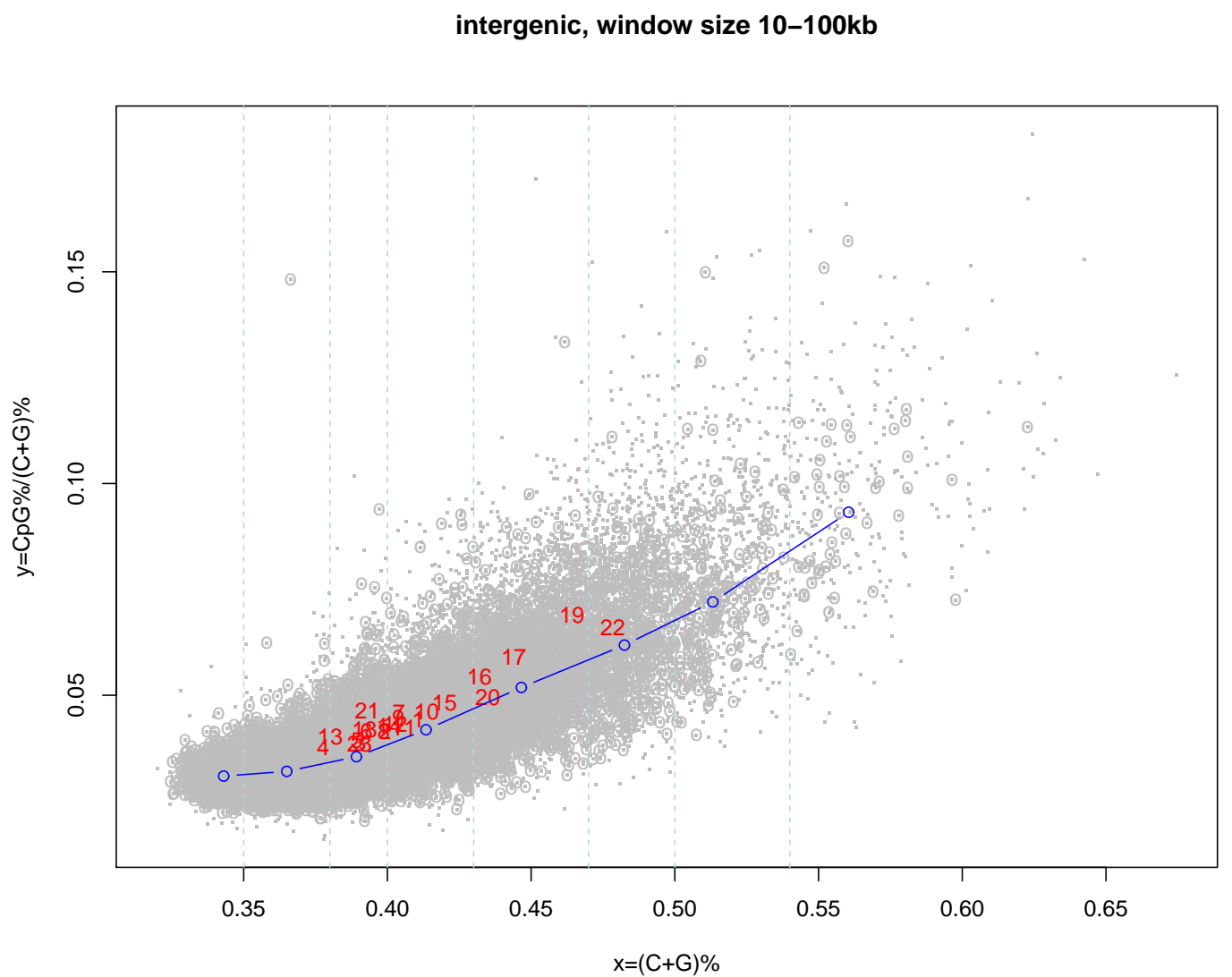

Figure 3: Each point is an intergenic region in the human genome (regions longer than 100kb are split into multiple 100kb windows), where $\mathrm{x}$ is $\mathrm{G}+\mathrm{C}$ content, $\mathrm{y}$ is the $\mathrm{CpG} /(\mathrm{G}+\mathrm{C})$ proportion. Larger windows/regions $(>80 \mathrm{~kb})$ are represented by circles. The $\mathrm{x}=\mathrm{G}+\mathrm{C}$ is partitioned into 6 ranges, where the blue dots represent the bin average. The chromosome-level averages are indicated by red letters. 


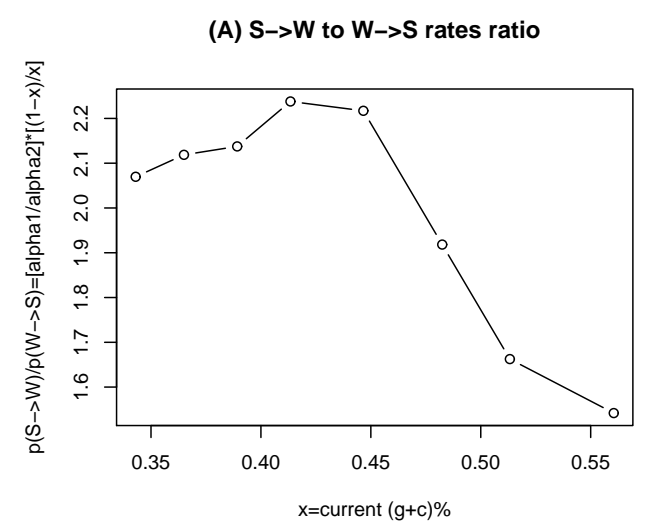

(C) limiting $\mathrm{CpG} /(\mathrm{G}+\mathrm{C})$

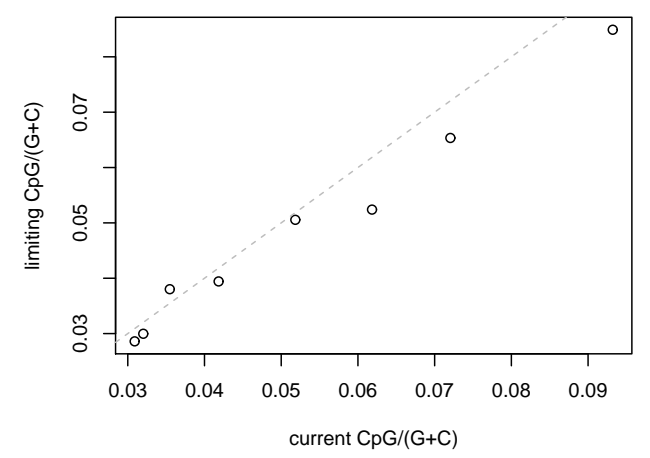

(B) limiting $(\mathrm{G}+\mathrm{C}) \%$

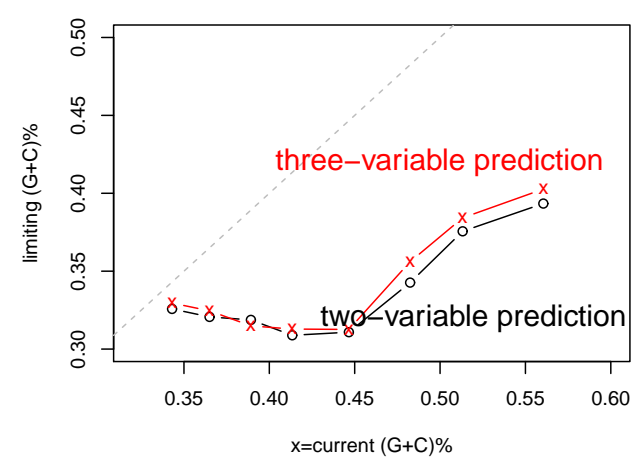

(D) $(G+C) \%$ in de novo bases

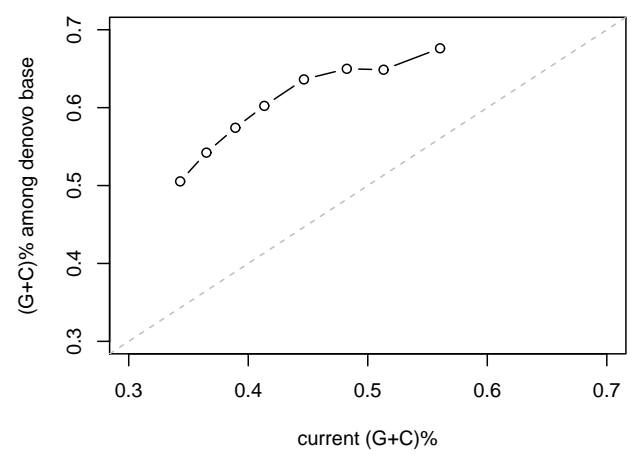

Figure 4: Evidence that current isochore structure might be maintained in the equilibrium configuration in neutral dynamics. Each bin represents a collection of de novo events in intergenic regions with specific $2 \mathrm{~kb}$ window $\mathrm{G}+\mathrm{C}$ content. The first five $\mathrm{G}+\mathrm{C}$ bin points contain 6000-7000 mutational events each, whereas the last 3 high $\mathrm{G}+\mathrm{C}$ bin points only 2000-3000 mutational events each. (A) $\frac{p_{S \rightarrow W}}{p_{W \rightarrow S}}$ (Eq. (3) $)$ or $\frac{\alpha_{1}(x)}{\alpha_{2}(x)} \cdot \frac{1-x}{x}$ (Eq. (4) ) as a function of current $2 \mathrm{~kb} \mathrm{G}+\mathrm{C}$ content; (B) limiting $\mathrm{G}+\mathrm{C}$ by Eq.(4) or Eq.(7) as a function of current $2 \mathrm{~kb}$ $\mathrm{G}+\mathrm{C}$ content; (C) limiting $\mathrm{CpG} /(\mathrm{G}+\mathrm{C})$ by Eq.(7) as a function of current 2kb CpG/(G+C); (D) proportion of $\mathrm{G}+\mathrm{C}$ among bases which have experienced de novo mutation. The grey lines in (B), (C), (D) represent slope $=1$ diagonal lines. 


\begin{tabular}{c|c|cccccc|cc|ccc}
\hline type & $\mathrm{n}$ & $\mathrm{n}(\mathrm{WS})$ & $\mathrm{n}\left(\mathrm{WS}_{p}\right)$ & $\mathrm{n}(\mathrm{SW})$ & $\mathrm{n}\left(\mathrm{SS}_{p}\right)$ & $\mathrm{n}\left(\mathrm{S}_{p} \mathrm{~W}\right)$ & $\mathrm{n}\left(\mathrm{S}_{p} \mathrm{~S}\right)$ & $\alpha_{1}$ & $\alpha_{2}$ & $\beta_{1}$ & $\beta_{2}$ & $\beta_{3}$ \\
\hline intron & 58824 & 14100 & 5926 & 18051 & 1108 & 11646 & 523 & 0.597 & 0.403 & 0.434 & 0.326 & 0.241 \\
intergenic & 40375 & 9813 & 4018 & 13277 & 732 & 6705 & 303 & 0.591 & 0.409 & 0.423 & 0.302 & 0.274 \\
missense & 3235 & 493 & 272 & 875 & 70 & 1083 & 59 & 0.719 & 0.281 & 0.553 & 0.298 & 0.150 \\
stream & 4832 & 1041 & 513 & 1505 & 123 & 991 & 44 & 0.616 & 0.384 & 0.449 & 0.294 & 0.257 \\
synonymous & 1336 & 167 & 112 & 431 & 24 & 508 & 10 & 0.771 & 0.229 & 0.623 & 0.232 & 0.145 \\
UTR & 1514 & 359 & 136 & 464 & 34 & 307 & 32 & 0.609 & 0.391 & 0.449 & 0.337 & 0.214 \\
non-coding & 634 & 98 & 68 & 169 & 19 & 171 & 16 & 0.672 & 0.328 & 0.506 & 0.282 & 0.213 \\
stop & 149 & 3 & 1 & 61 & 0 & 66 & 0 & 0.969 & 0.031 & 0.940 & 0.046 & 0.006 \\
splice & 81 & 4 & 16 & 42 & 0 & 1 & 0 & 0.683 & 0.317 & 0.058 & 0.006 & 0.936 \\
\hline
\end{tabular}

Table 1: Statistics of the de novo mutational events used: type: 9 functional groups are used by simplifying the original 18 groups; $n$ : number of mutational events; $n(W S)$ : number of de novo mutation from $W$ (A or $\mathrm{T}$ ) to $S$ (G or $\mathrm{C}$ ) bases outside a CpG-containing triplet; $n(W S p)$ : number of de novo mutation from $W$ to $S$ bases within a CpG-containing triplet ; $n(S W)$ : number of de novo mutation from $S$ (non-CpG) to $W$ bases; $n(S S p)$ : number of de novo mutation from $S$ (non-CpG) to $S$ (CpG containing) bases; $\alpha_{1}, \alpha_{2}$ : defined in Eq.(33), satisfying $\alpha_{1}+\alpha_{2}=1 ; \beta_{1}, \beta_{2}, \beta_{3}$ : defined in Eq.(7), satisfying $\beta_{1}+\beta_{2}+\beta_{3}=1$. 
(i) de novo mutation in intergenic regions

\begin{tabular}{|c|c|c|c|c|c|c|c|c|c|c|c|c|}
\hline $\mathrm{G}+\mathrm{C}$ range & $\mathrm{n}$ & $\mathrm{n}(\mathrm{WS})$ & $\mathrm{n}\left(\mathrm{WS}_{p}\right)$ & $\mathrm{n}(\mathrm{SW})$ & $\mathrm{n}\left(\mathrm{SS}_{p}\right)$ & $\mathrm{n}\left(\mathrm{S}_{p} \mathrm{~W}\right)$ & $\mathrm{n}\left(\mathrm{S}_{p} \mathrm{~S}\right)$ & $\alpha_{1}$ & $\alpha_{2}$ & $\beta_{1}$ & $\beta_{2}$ & $\beta_{3}$ \\
\hline $0.1-0.35$ & 6449 & 2008 & 558 & 2075 & 78 & 698 & 27 & 0.519 & 0.481 & 0.355 & 0.335 & 0.310 \\
\hline $0.35-0.38$ & 7288 & 2045 & 724 & 2361 & 109 & 1011 & 31 & 0.549 & 0.451 & 0.382 & 0.320 & 0.299 \\
\hline $0.38-0.40$ & 6890 & 1775 & 639 & 2383 & 124 & 905 & 37 & 0.577 & 0.423 & 0.401 & 0.289 & 0.310 \\
\hline $0.40-0.43$ & 6125 & 1365 & 620 & 2039 & 114 & 1092 & 48 & 0.612 & 0.388 & 0.443 & 0.287 & 0.270 \\
\hline $0.43-0.47$ & 6315 & 1285 & 645 & 2193 & 128 & 1260 & 45 & 0.641 & 0.359 & 0.473 & 0.267 & 0.260 \\
\hline $0.47-0.76$ & 7308 & 1335 & 832 & 2226 & 179 & 1739 & 115 & 0.647 & 0.353 & 0.480 & 0.278 & 0.242 \\
\hline $0.47-0.5$ & 3136 & 610 & 330 & 985 & 65 & 696 & 38 & 0.641 & 0.359 & 0.476 & 0.285 & 0.239 \\
\hline $0.5-0.54$ & 1964 & 373 & 230 & 602 & 49 & 455 & 28 & 0.637 & 0.363 & 0.469 & 0.279 & 0.252 \\
\hline $0.54-0.76$ & 2208 & 352 & 272 & 639 & 65 & 588 & 49 & 0.663 & 0.337 & 0.496 & 0.265 & 0.239 \\
\hline \multicolumn{13}{|c|}{ (ii) equilibrium $(\mathrm{G}+\mathrm{C}) \%$ andf $\mathrm{CpG} \%$} \\
\hline $\mathrm{G}+\mathrm{C}$ range & \multicolumn{3}{|c|}{$(\mathrm{G}+\mathrm{C}) \% \quad \mathrm{CpG} \% /(\mathrm{G}+\mathrm{C}) \%$} & \multicolumn{5}{|c|}{ eq $(\mathrm{G}+\mathrm{C})$ by Eq. (4) eq $(\mathrm{G}+\mathrm{C})$ by Eq. (7) } & \multicolumn{4}{|c|}{ eq $\mathrm{CpG} /(\mathrm{G}+\mathrm{C})$ by Eq. (77) } \\
\hline $0.1-0.35$ & \multicolumn{2}{|c|}{0.343} & \multicolumn{2}{|l|}{0.0309} & \multicolumn{2}{|c|}{0.326} & \multicolumn{2}{|c|}{0.329} & \multicolumn{4}{|c|}{0.0286} \\
\hline $0.35-0.38$ & \multicolumn{2}{|c|}{0.365} & \multicolumn{2}{|l|}{0.0320} & \multicolumn{2}{|l|}{0.321} & \multicolumn{2}{|c|}{0.325} & \multicolumn{4}{|c|}{0.0300} \\
\hline $0.38-0.40$ & \multicolumn{2}{|c|}{0.389} & \multicolumn{2}{|l|}{0.0355} & \multicolumn{2}{|l|}{0.319} & \multicolumn{2}{|c|}{0.315} & \multicolumn{4}{|c|}{0.0380} \\
\hline $0.40-0.43$ & \multicolumn{2}{|c|}{0.413} & \multicolumn{2}{|l|}{0.0418} & \multicolumn{2}{|l|}{0.309} & 0.3 & & & & 394 & \\
\hline $0.43-0.47$ & 0.447 & & 0.0518 & & 0.311 & & 0.3 & & & & 506 & \\
\hline $0.47-0.76$ & 0.496 & & 0.0667 & & 0.350 & & 0.3 & & & & 586 & \\
\hline $0.47-0.5$ & 0.482 & & 0.0618 & & 0.343 & & 0.3 & & & & 524 & \\
\hline $0.5-0.54$ & 0.513 & & 0.0721 & & 0.376 & & 0.38 & & & & 653 & \\
\hline $0.54-0.76$ & 0.560 & & 0.0932 & & 0.393 & & 0.44 & & & & 849 & \\
\hline
\end{tabular}

Table 2: Statistics of intergenic de novo mutations partitioned into their $2 \mathrm{~kb}$ window $\mathrm{G}+\mathrm{C}$ content range $(6$ bins for rough equal number partition, and last bin further split into three bins): (i) see the caption of Table 1 . (ii) predicted limiting equilibrium by current $\mathrm{G}+\mathrm{C}$ content using Eq.(4), or by both current $\mathrm{G}+\mathrm{C}$ and current $\mathrm{CpG} /(\mathrm{G}+\mathrm{C})$ using Eq. (17). 